\title{
Clinically important health utility gains in cochlear implant recipients
}

\author{
Lida Müller ${ }^{1}$, Petra Graham², Jasmin Kaur $^{3}$, Josie Wyss ${ }^{3}$, Paula Greenham ${ }^{4}$, and Chris \\ James $^{5}$ \\ ${ }^{1}$ Tygerberg Hospital - Stellenbosch University \\ ${ }^{2}$ Macquarie University \\ ${ }^{3}$ Cochlear AG \\ ${ }^{4}$ Greenham consulting \\ ${ }^{5}$ Cochlear France SAS
}

August 28, 2020

\begin{abstract}
Objectives: Cochlear implantation can restore access to sound and speech understanding in subjects with substantial hearing loss where acoustic hearing aids do not. The Health Utilities Index Mark III (HUI3) is commonly used to measure health utility across all types of intervention and is sensitive to changes in hearing. In the current study we analysed for factors predicting clinically important gains in HUI3 scores in adult Nucleus cochlear implant recipients, implanted in a single centre. Design: Retrospective analysis of data collected in an observational study. Demographic and other baseline parameters were analysed for their association with gains in HUI3 scores. Participants: One-hundred and thirty-seven adult recipients of Nucleus cochlear implants who had at least one-year follow-up. Main Outcome Measures: HUI3 scores and speech, spatial, quality (SSQ) scale scores were collected at baseline before device activation and one-year after. Difference scores were computed. Clinically important gain was defined as 0.1 point on the HUI3 scale. Results: Baseline telephone use and baseline HUI3 hearing, speech and emotion attribute levels were significantly associated with clinically important gains in HUI3 scores. However, SSQ scores increased significantly with or without clinically important gains in HUI3 scores. Conclusion: Those subjects who were unable to use the telephone prior to cochlear implantation were one-and-a-half times more likely to obtain a clinically important gain in health utility. Those subjects who were unhappy, or experienced difficulties communicating with strangers were twice or more likely to obtain a clinically important gain in HUI3 scores compared to those who did not.
\end{abstract}

\section{Introduction}

The success of a health care intervention is often measured by its impact on the patient's quality of life. This provides a tool for comparing the success of different treatments, regardless of the condition they are targeting (Swan et al., 2012). Standardised measures of health-related quality of life are used to provide a health utility score. This can then be used to calculate any gains in quality adjusted life years and assess a treatment's cost effectiveness (Foteff et al., 2016; Saunders et al., 2016). Three generic preference-based questionnaires are commonly used to provide a measure of health utility; EuroQol 5 dimensions (EQ-5D), Health Utility Index 3 (HUI3) and Short-form 6 dimensions (SF-6D) (Herdman et al., 2011, Feeny et al., 2002, Brazier et al., 2002). Of these, only the HUI3 is sensitive to changes in hearing and is recommended as the questionnaire of choice for studies evaluating hearing treatments (Summerfield et al., 2019; Arnoldner et al., 2014; Yang et al., 2013).

The HUI3 consists of 15 questions addressing eight individual health attributes: vision, hearing, speech, ambulation, dexterity, emotion, cognition and pain. Each health attribute has 5 or 6 levels and a weighted 
health utility score assigned to each level which varies by attribute. These scores are then combined to give a measure of the overall health utility (Feeny et al., 2002). Scores range from zero (dead) to one (perfect health) or even a negative score indicating a state worse than dead. HUI3 scores of less than 0.7 are considered to indicate a severe disability, between 0.7 and 0.88 a moderate disability, and 0.89 or better a mild or no disability (Feng et al., 2009). An increase in score of at least 0.03 is thought to represent a noticeable improvement in quality of life for a patient (Feeney et al. 1995) and an increase of 0.1, a minimum clinically important change (UK Cochlear Implant Study Group, 2004). In studies looking at the benefits of fitting acoustic hearing aids, gains in health utility ranged from 0.06 (Barton et al., 2004) to 0.12 (Grutters et al., 2007; Swan et al., 2012). However, scores remain in the moderate to severe disability category even after treatment (Yan et al., 2009). Cochlear implantation (CI) provides an alternative treatment for those with severe to profound hearing loss with overall greater gains in HUI3 scores of $\sim 0.20$ (range 0.05 to 0.4 , Crowson et al., 2017).

In South Africa approximately $83 \%$ of the population depends on public health and $17 \%$ on the private health care market financed by the medical industry. Funding is provided for CIs on an ad hoc basis from the public health sector through Tygerberg Hospital, with large waiting lists for adult candidates. In countries where access to CI is limited, establishing who will benefit most is one way of prioritising treatment. For cochlear implantation, however, outcomes are difficult to predict from preoperative measures (Lazard et al., 2012). An actuarial approach acknowledges this variability in outcomes and expresses the a priori odds of getting a better result post treatment (UK CI study group, 2004). Up until now the definition of a 'better result' has been a greater improvement in speech perception outcomes. Improvements in quality of life, however, have consistently been shown to be independent of audiological performance (Crowson et al., 2017), and therefore may be more meaningful for patients.

Few studies have looked at whether the change in HUI3 scores resulting from implantation can be predicted using preoperative factors. The aim of this study was to determine which baseline factors may significantly influence clinically important gains in HUI3. We considered a gain of [?]0.1 in HUI3 multi-attribute score as clinically important based on the literature (UK CI study group, 2004). The intention was to provide a guide for clinicians when counselling potential patients about the benefits of a CI and to help them prioritize candidates in clinics with large waiting lists and limited resources.

\section{Methods}

Data were gathered via a voluntary online international observational registry for hearing implants initiated by [removed for blind peer review]. Data were collected in accordance with the Declaration of Helsinki. Ethics approval was obtained from the Health Research Ethics Committee [removed for blind peer review]. Each subject provided their written informed consent for their data to be included in the registry. The reporting here complies with the RECORD statement (Benchimol et al., 2015).

\section{Subjects}

We extracted data for all adult unilateral Nucleus CI recipients ( $>17$ years old) implanted at [removed for blind peer review] Cochlear Implant Unit between 2012 and 2019. Baseline data were collected prior to activation of the processor after a decision to implant had been made. we focus on the gain from baseline to 1-year post-activation. Self-assessments were completed by the recipient either online or on paper and then entered into the database by a representative of the clinic.

\section{Outcome measures}

The Health Utilities Index Mark 3 (HUI3), a generic measure of health utility gains (Feeny et al., 1995) was the primary measure. Change from baseline to first follow-up in the HUI multi-attribute above or below 0.1 units was the primary outcome in this study.

Table 1 illustrates the structure of the HUI3 hearing attribute levels (Feeny et al., 2012). Levels are designated based on the combined responses to two questions about hearing. Levels 1 and 2, as the highest/best levels indicate that the respondent does not always need a hearing aid to hear what is said, whereas the lower 
levels of performance 3-5 require use of a hearing aid (or other assistive hearing device) and report the level of performance with that device. Level 6 indicates unable to hear anything at all, even with hearing aids. Furthermore, only levels 1-3 indicate that respondents can hear what is said in more difficult situations. We have designated the categories "able to hear" and "unable to hear" to the hearing attribute levels 1-3 and 4-6 for the purposes of the discussion. Obtaining level 3 (or greater) means that the intervention was essentially successful in restoring the capacity to hear what is said, even in more challenging situations, so long as a hearing aid, or in this case CI, is being used.

Weighting scores for given levels of each health attribute are used to produce a HUI3 "total" or multi-attribute score combining all eight. As in table 1, some transitions between adjacent hearing attribute levels (e.g. for hearing 6 to 5 , and 4 to 3) may possibly result in changes of [?]0.1 in the combined HUI3 multi-attribute scores, but often at least two levels need to be gained for this to be the case. This is a property of the way the HUI3 was constructed.

Data were also collected for the Speech Spatial Qualities (SSQ) scale (Gatehouse and Noble, 2004). This is a disease specific scale which aims to evaluate the subject's speech understanding in quiet and noise, spatial perception and the clarity, separation, and identification of sounds. Changes of at least 1.0 units on the SSQ subscales indicates a clinically relevant change (Noble and Gatehouse, 2006). Additional information was collected about each subject's hearing history and demographics.

\section{TABLE 1 HERE}

\section{Statistics}

Because interest was in those who gained at least the clinically important difference (MCID) of 0.1 HUI3 multi-attribute score units, change in HUI3 multi-attribute score was dichotomized at this value with a 1 representing achievement of at least the MCID and 0 otherwise. Fisher's exact test was used to determine the association between the dichotomized HUI3 multi-attribute change score and variables of interest. Table 3 shows the variables considered with variables summarized using count and percent. Levels for the eight attributes were dichotomized into worse/better outcomes so that counts in each category were sufficiently large to detect significant differences between proportions, if they existed. Paired t-tests were used to examine change over time from baseline to year 1 follow-up. P-values less than 0.05 were considered significant. All analyses were conducted in $\mathrm{R}^{4}$.

\section{Results}

\section{Gains in HUI3}

175 subjects had baseline HUI data, 137 of those had follow-up at 1 year, 97 at year 2 and 75 at year 3 . A statistically significant and clinically relevant improvement in HUI3 multi-attribute scores from baseline was seen for the group at year 1 (mean change $=0.16,95 \%$ CI:0.11-0.21; $<<0.001$ ), which was maintained at years 2 and 3 (Figure 1, left). As can be seen in the right panel of Figure 1, preoperatively $65 \%$ were at generic "unable to hear" levels (4-5-6). Post-operatively this situation was more than reversed with $77 \%$ at generic "able to hear" levels (1-2-3).

\section{FIGURE 1 HERE}

A closer look at the individual changes in hearing attribute levels is available in Table 2; comparing preoperative with 1-year postoperative for 137 subjects with both data points. Two contingencies contributed most often to [?]0.1 gain in HUI3 multi-attribute score; those at level 5 and 6 preoperatively, or generically "unable to hear", moving to levels 1-3 postoperatively. Table 2 also indicates that about one third $(\mathrm{n}=46,33.6 \%)$ of subjects had no change in hearing attribute level, with by far most of those starting and finishing at level 3. Overall $74(54 \%)$ subjects fell above the dividing line for significant hearing attribute level increase, and 63 (46\%) below.

Based on the mechanism for increase in HUI3 scores elucidated in Table 1, the transitions in hearing attribute level (Table 2) contributed to 74 of the total 81/137 (59\%) subjects with [?]0.1 HUI3 gain. 


\section{TABLE 2 HERE}

\section{Baseline predictors of clinically important HUI3 gains}

In Table 3 we present the results of the factor analysis of baseline variables on the bivariate outcome for less than or greater than a 0.1-point gain in HUI3 score.

Subjects unable to use the telephone preoperatively were one-and-a-half times more likely to obtain [?]0.1 gain in HUI3 scores (odds ratio OR 1.47); those reporting difficulty to be understood by strangers or worse twice as likely (OR 2.27); and those reporting being unhappy or worse two-and-a-half times more likely (OR 2.53). There was no effect of age, sex or duration of deafness.

\section{TABLE 3 HERE}

\section{Gains in SSQ scores according to gain in HUI3 scores}

SSQ scores improved significantly from baseline to 1-year both for who obtained clinically important gains in HUI3 score (mean SSQ change 3.18, 95\%CI:2.73-3.62, p<0.001) and for those who did not (mean SSQ change $2.43,95 \%$ CI:1.98-2.88, $\mathrm{p}<0.001)$.

\section{Discussion}

Baseline scores placed the majority of the sample in the severe disability category of HUI3 multi-attribute score (Figure 1, left) levels i.e. where at least one attribute is at a reduced level of function that cannot be corrected and prevents many activities (Feng et al., 2009). This is typical for a cohort of hearing-impaired individuals with sensorineural hearing loss (Summerfield et al., 2019; Swan et al., 2012; Barton et al 2012). After implantation, there was a significant gain in HUI3 scores at one year which was maintained at 2 and 3 years. At least $50 \%$ of the subjects had moved into the moderate disability category (Figure 1, left), where at least one attribute is at a reduced level of function that cannot be corrected and/or prevents some activities. However, we see that most subjects reached level 3 on the hearing attribute scale. This level describes functional hearing with the use of a "hearing aid" (in this case a CI).

Of the factors considered in the logistic regression analysis telephone use and the speech and emotion attributes on HUI3 were significant. Those who did not use the telephone at baseline were 1.5 times more likely to get a clinically meaningful gain on the HUI3 from implantation. As expected, subjects in the poor hearing category at baseline, (hearing attribute levels 4,5 or 6 ) were also more likely not to use the telephone (OR 1.52). Thus, telephone use is an easy parameter to use for the screening of potential adult CI candidates presenting to the audiology clinic. With poor access to adult hearing screening in many countries, the capability to use the telephone use would provide a simple criterion for urgent referral for cochlear implant assessment. Those who reported that strangers were unable to understand them, or even poorer verbal communication, also had a higher chance of clinically significant gain in health utility. Improved overall verbal communication due to better hearing and other rehabilitation following CI may have contributed to this effect. Those who were unhappy prior to implant were also more likely to show a meaningful gain in the HUI3, probably due to the implant and rehabilitation process improving their communication and thus emotional state.

The Fisher's exact test showed that there was also a non-random association between a meaningful gain on the HUI3 and the hearing single attribute. Not surprisingly, those subjects with poor baseline hearing were more likely to gain more than 0.1 points. The SSQ was still sensitive to changes in hearing brought about by CI, even in the group with no clinically important gain in HUI3. For that group the mean improvement of 2.43 on the SSQ scale was highly clinically significant.

\section{Limitations}

This was an observational study and enrolment bias may be present in the sample. Investigators were instructed to offer participation to all consecutively implanted adult and adolescent Nucleus@ implant recipients. However, recipients who were more motivated which may influence outcomes may have been more 
likely to agree to participate. As self-assessment was required, those with severe comorbidities and those who are unable to read were less likely to be included. Baseline evaluations were made between the day of the surgery and the first fitting, which may result in a recall bias.

\section{Conclusions}

After cochlear implantation there was a statistically significant group gain in HUI3 scores at one year which was maintained at years 2 and 3. Those who did not use the telephone at baseline were 1.5 times more likely to get a clinically important gain of [?]0.1 in HUI3 multi-attribute score from cochlear implantation. Those with lower hearing, speech or emotion HUI3 single-attribute levels prior to implantation were also more likely to obtain clinically important gains in HUI3 scores. An incapacity to use the telephone may be a useful biomarker for cochlear implant candidacy prior to formal audiological evaluation.

For those the subjects who obtained less than 0.1-point gain in HUI3, this could often be attributed to a lack of change in the hearing attribute level. However, mean hearing performance measured by the SSQ still increased significantly for that group after CI. The HUI3 scale stands in good stead as a health-utility instrument to measure the benefits of cochlear implantation for those with severe handicap due to hearing impairment, but lacks sensitivity to changes in hearing performance compared with a disease specific measure such as the Speech, Spatial Qualities (SSQ) scale.

\section{References}

Arnoldner C, Lin VY, Bresler R, Kaider A, Kuthubutheen J, Shipp D, Chen JM. Quality of life in cochlear implantees: comparing utility values obtained through the Medical Outcome Study Short-Form Survey-6D and the Health Utility Index Mark 3. Laryngoscope. 2014 Nov;124(11):2586-90. doi: 10.1002/lary.24648. Epub 2014 Aug 11.

Barton GR, Bankart J, Davis AC, Summerfield QA. (2004). Comparing Utility Scores before and after hearing-aid provision: Results according to the EQ-5D, HUI3 and SF-6D. Applied Health Economics and Health Policy, 3(2), 103-105.

Benchimol E.I., Smeeth L., Guttmann A., et al. (2015) The REporting of studies Conducted using Observational Routinely-collected health Data (RECORD) Statement. PLOS Med. 12, e1001885.

Brazier J., Roberts J. \& Deverill M. (2002) The estimation of a preference-based measure of health from the SF-36. J. Health Econ. 21 , 271-292.

Crowson M, G, Semenov Y, R, Tucci D, L, Niparko J, K: Quality of Life and Cost-Effectiveness of Cochlear Implants: A Narrative Review. Audiol Neurotol 2017;22:236-258. doi: 10.1159/000481767

Herdman M., Gudex C., Lloyd A., et al. (2011) Development and preliminary testing of the new five-level version of EQ-5D (EQ-5D-5L). Quali life res $20,1727-36$.

Feeny D, Furlong W, Boyle M, Torrance GW: Multi-attribute health status classification systems: Health Utilities Index. Pharmacoeconomics 1995; 7: 490-502.

Feeny D, Furlong W, Torrance GW, Goldsmith CH, Zhu Z, DePauw S, Denton M, Boyle M Multi-attribute and single-attribute utility functions for the health utilities index mark 3 system. Med Care. 2002 Feb; 40(2):113-28.

Feeny D, Huguet N, McFarland BH, Kaplan MS, Orpana H, Eckstrom E. Hearing, mobility, and pain predict mortality: a longitudinal population-based study. J Clin Epidemiol . 2012;65(7):764-777. doi:10.1016/j.jclinepi.2012.01.003

Feng Y, Bernier J, McIntosh C, Orpana H, "Validation of disability categories derived from Health Utilities Index Mark 3 scores", Statistics Canada, 2009, Health Reports, Vol. 20, No. 2:43-50. Catalogue number 82-003-X. 
Foteff C, Kennedy S, Milton AH, Deger M, Payk F, Sanderson G. Cost-Utility Analysis of Cochlear Implantation in Australian Adults. Otol Neurotol. 2016 Jun;37(5):454-61. doi: 10.1097/MAO.0000000000000999.

Gatehouse S, Noble W. The Speech, Spatial and Qualities of Hearing Scale (SSQ). Int J Audiol. 2004 Feb;43(2):85-99. PubMed PMID: 15035561; PubMed Central PMCID: PMC5593096.

Grutters JP, Joore MA, van der Horst F, Verschuure H, Dreschler WA, Anteunis LJ. Choosing between measures: comparison of EQ-5D, HUI2 and HUI3 in persons with hearing complaints. Qual Life Res . 2007;16(8):1439-1449. doi:10.1007/s11136-007-9237-x

Lazard DS, Vincent C, Venail F, Van de Heyning P, Truy E, Sterkers O, Skarzynski PH, Skarzynski H, Schauwers K, O'Leary S, Mawman D, Maat B, Kleine-Punte A, Huber AM, Green K, Govaerts PJ, Fraysse B, Dowell R, Dillier N, Burke E, Beynon A, Bergeron F, Başkent D, Artières F, Blamey PJ. Pre-, per- and postoperative factors affecting performance of postlinguistically deaf adults using cochlear implants: a new conceptual model over time. PLoS One. 2012;7(11):e48739. doi: 10.1371/journal.pone.0048739. Epub 2012 Nov 9. PubMed PMID: 23152797; PubMed Central PMCID: PMC3494723.

Lenarz T, Muller L, Czerniejewska-Wolska H, Varela HV, Dotú CO, Durko M, ... Wyss J. (2017). Patientrelated benefits for adults with cochlear implantation: a multicultural longitudinal observational study. Audiology and Neurotology, 22(2), 61-73. https://doi.org/10.1159/000477533

Noble W, Gatehouse S. (2006) Effects of bilateral versus unilateral hearing aid fitting on abilities measured by the Speech, Spatial, and Qualities of Hearing Scale (SSQ). Int. J. Audiol. 45, 172-81.

R Core Team (2017) R: A Language and Environment for Statistical Computing.

Saunders JE, Francis HW, Skarzynski PH. Measuring Success: Cost-Effectiveness and Expanding Access to Cochlear Implantation. Otol Neurotol. 2016 Feb;37(2):e135-40. doi: 10.1097/MAO.0000000000000942.

Summerfield AQ, Barton GR. Sensitivity of EQ-5D-3L, HUI2, HUI3, and SF-6D to changes in speech reception and tinnitus associated with cochlear implantation. Quality of Life Research 2019; 28:1145-1154 https://doi.org/10.1007/s11136-018-2070-6

Swan IR, Guy FH, Akeroyd MA. Health-related quality of life before and after management in adults referred to otolaryngology: a prospective national study. Clin Otolaryngol . 2012;37(1):35-43. doi:10.1111/j.17494486.2011.02433.x

UK Cochlear Implant Study Group. Criteria of candidacy for unilateral cochlear implantation in postlingually deafened adults III: prospective evaluation of an actuarial approach to defining a criterion. Ear Hear. 2004 Aug;25(4):361-74. PubMed PMID: 15292776.

Yang Y, Longworth L, Brazier J. An assessment of validity and responsiveness of generic measures of healthrelated quality of life in hearing impairment. Qual Life Res . 2013;22(10):2813-2828. doi:10.1007/s11136013-0417-6

Table 1. HUI3 hearing attribute levels and descriptors from Feeny et al. (2002). The third column indicates binary categories of "able to hear" or "unable to hear" that we define here. The final column indicates upward transitions in adjacent levels (shaded) that could result in [?]0.1 HUI3. All upward transitions of greater than one level potentially result in [?]0.1 HUI3 gain. 


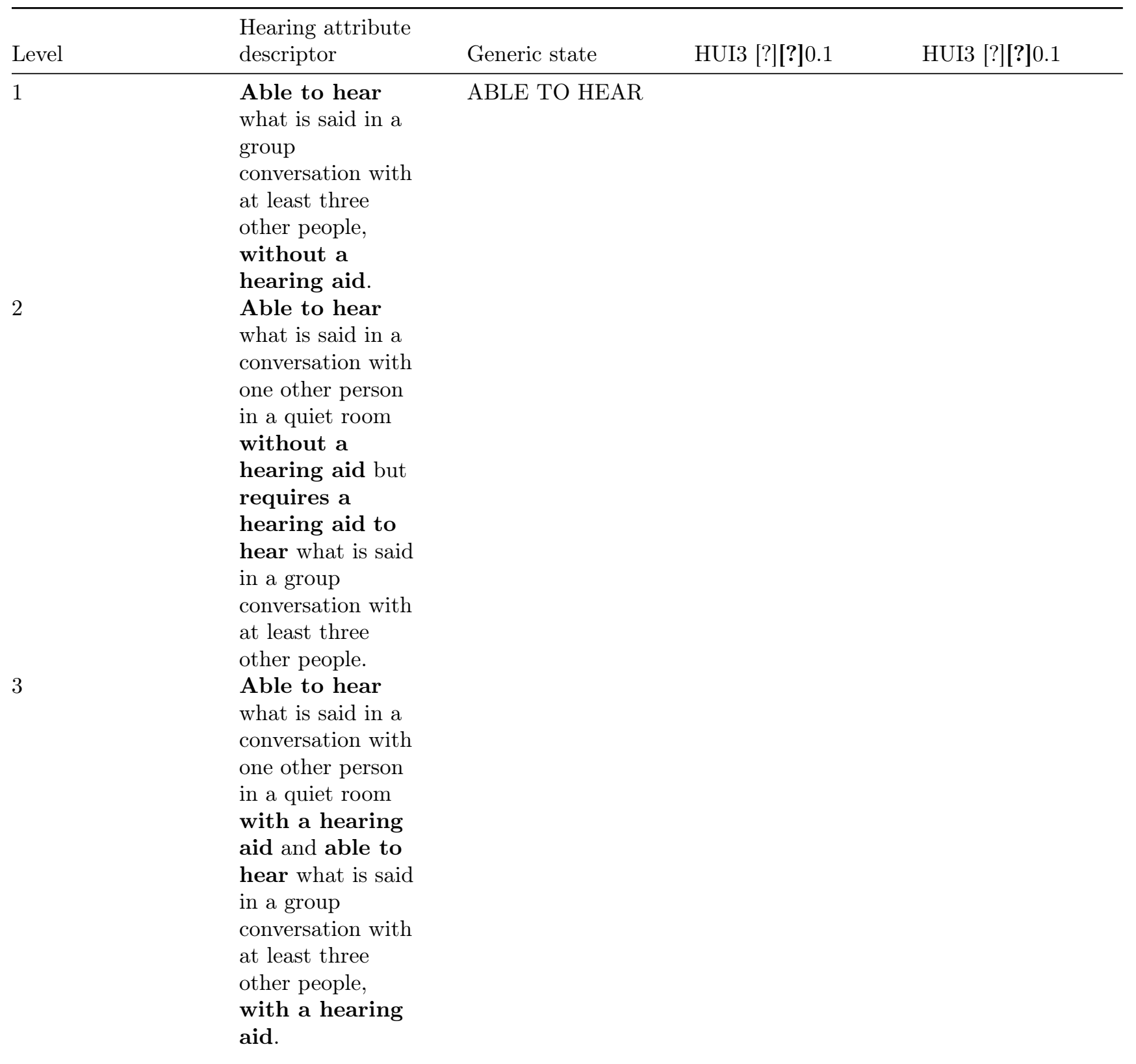




\begin{tabular}{|c|c|c|c|c|}
\hline Level & $\begin{array}{l}\text { Hearing attribute } \\
\text { descriptor }\end{array}$ & Generic state & HUI3 [?][?]0.1 & HUI3 [?][?]0.1 \\
\hline 4 & $\begin{array}{l}\text { Able to hear } \\
\text { what is said in a } \\
\text { conversation with } \\
\text { one other person } \\
\text { in a quiet room } \\
\text { without a } \\
\text { hearing aid, but } \\
\text { unable to hear } \\
\text { what is said in a } \\
\text { group } \\
\text { conversation with } \\
\text { at least three } \\
\text { other people even } \\
\text { with a hearing } \\
\text { aid. }\end{array}$ & $\begin{array}{l}\text { UNABLE TO } \\
\text { HEAR }\end{array}$ & & \\
\hline 5 & $\begin{array}{l}\text { Able to hear } \\
\text { what is said in a } \\
\text { conversation with } \\
\text { one other person } \\
\text { in a quiet room } \\
\text { with a hearing } \\
\text { aid but unable } \\
\text { to hear what is } \\
\text { said in a group } \\
\text { conversation with } \\
\text { at least three } \\
\text { other people even } \\
\text { with a hearing } \\
\text { aid. }\end{array}$ & & & \\
\hline 6 & $\begin{array}{l}\text { Unable to hear } \\
\text { at all. }\end{array}$ & & & \\
\hline
\end{tabular}

Table 2. Cross-tabulation of the number of subjects at each hearing attribute level at baseline and postoperatively. Above the bold line indicates contingencies which would potentially result in [?]0.1 gain in HUI3 multi-attribute score as indicated in Table 1.

\begin{tabular}{|c|c|c|c|c|c|c|c|}
\hline \multicolumn{7}{|l|}{ Postoperative } & 1 \\
\hline & 2 & 2 & 1 & 0 & 1 & 0 & 0 \\
\hline & 3 & 24 & 34 & 2 & 32 & 1 & 3 \\
\hline & 4 & 0 & 1 & 0 & 0 & 0 & 1 \\
\hline & 5 & 7 & 9 & 0 & 3 & 1 & 0 \\
\hline & 6 & 4 & 4 & 0 & 2 & 0 & 0 \\
\hline & & 6 & 5 & 4 & 3 & 2 & 1 \\
\hline
\end{tabular}




\begin{tabular}{lllllll}
\hline $\begin{array}{l}\text { Postoperative } \\
\text { level }\end{array}$ & 1 & 1 & 1 & & & \\
& & & & & \\
& Baseline & Baseline & Baseline & Baseline & Baseline & Baseline \\
& hearing & hearing & hearing & hearing & hearing \\
& attribute & attribute & attribute & attribute & attribute \\
& level & level & level & level & level level \\
\hline
\end{tabular}

Table 3. Count (percentage) of those who did or did not obtain a clinically important gain in HUI3 multi-attribute score (p-value from Fisher's exact test). Items in italics represent unreliable figures due to very small counts in some categories. Items in bold were significant at the $5 \%$ level.

P-values from Fisher's exact tests

Categorical covariate: $\mathrm{n}(\%)$

HUI gain $<0.1 \mathrm{n}=56$

HUI gain [?]0.1 $\mathrm{n}=81$

$\mathrm{P}$-value

Female

$37(66.1)$

$52(64.2)$

0.857

Right hand side implant

$44(78.6)$

$53(65.4)$

0.126

Implant side imaging indicates anomaly

$6(11.1)$

$6(7.4)$

0.542

Language Afrikaans English Other

18 (32.1) 26 (46.4) 12 (21.4)

16 (19.8) 54 (66.7) 11 (13.6)

0.061

Additional handicaps

$3(5.4)$

$1(1.2)$

0.305

Tinnitus present at baseline 
$26(46.4)$

44 (54.3)

0.389

Dizziness present at baseline

$32(57.1)$

$44(54.3)$

0.861

Onset of deafness Progressive Sudden

39 (70.9) 16 (29.1)

$60(74.1) 21(25.9)$

0.699

Hearing loss degree (implant side) Profound Not profound

44 (78.6) 12 (21.4)

$72(88.9) 9(11.1)$

0.147

Hearing loss degree (contralateral) Profound Not profound

43 (76.8) 13 (23.2)

$65(80.2) 16(19.8)$

0.674

Duration of deafness [?]13 years 14-24 years 25-37 years $38+$ years

$11(0.20) 17(0.31) 15(0.27) 12(0.22)$

$26(0.32) 21(0.26) 18(0.22) 16(0.20)$

0.481

Hearing aid use in the contralateral ear at baseline

$18(32.1)$

33 (40.7)

0.489

Age group 18-34 35-44 45-54 55-64 65-91

15 (26.8) 8 (14.3) 8 (14.3) 8 (14.3) 17 (30.4)

9 (11.1) 16 (19.8) 17 (21.0) 15 (18.5) 24 (29.6)

0.187

No telephone use

24 (42.9)

51 (63.0) 


\subsection{4}

Etiology*

0.560

Congenital onset of deafness

$15(27.3)$

$16(19.8)$

0.405

Usefulness of implant side hearing aid Marginally or not at all Moderately or more

28 (65.1) 15 (34.9)

48 (78.7) 13 (21.3)

0.178

Usefulness of contralateral hearing aid Marginally or not at all Moderately or more

$17(40.5) 25(59.5)$

$35(59.3) 24(40.7)$

0.072

HUI3 attribute vision Able to read with glasses or better Unable to read with glasses or worse

54 (96.4) 2 (3.6)

$77(95.1) 4(4.9)$

1.000

HUI3 attribute hearing Able to hear group with a hearing aid or better Unable to hear group with hearing aid or worse

33 (58.9) $23(41.1)$

$14(17.3) 67(82.7)$

$<0.001$

HUI3 attribute speech Able to be understood partially by strangers or better Unable to be understood by strangers or worse

49 (87.5) 7 (12.5)

58 (71.6) 23 (28.4)

0.035

HUI3 attribute ambulation Able to walk neighbourhood aided or unaided Walk short distances only or requires wheelchair

$55(98.2) 1(1.8)$

$77(95.1) 4(4.9)$

0.648

HUI3 attribute dexterity Limitations but independent or no limitations Not independent 
$54(96.4) 2(3.6)$

$81(100) 1(0)$

0.165

HUI3 attribute emotion Somewhat happy or better Somewhat unhappy or worse

$50(89.2) 6(10.7)$

$59(72.8) 22(27.2)$

0.030

HUI3 attribute cognition Forgetful but thinks clearly or not forgetful Forgetful with difficulties or can't remember at all

$51(91.1) 5(8.9)$

$70(86.4) 11(13.6)$

0.590

HUI3 attribute pain Mild or no pain; no activities prevented Moderate or more pain; activities prevented

$47(83.9) 9(16.1)$

$63(77.8) 18(22.2)$

0.513

*counts for each category shown in Appendix 1.

FIGURE LEGEND

Figure 1. HUI3 multi-attribute scores (left) and proportions of subjects at each hearing attribute level (right) at baseline and at 1, 2, and 3 years post implant. Boxes (left) show quartile values and the line the median value. Whiskers indicate the minimum and maximum value within 1.5 times the interquartile range and circles any outliers. We note on the right that level 1 is the best, and level 6 is the worst.
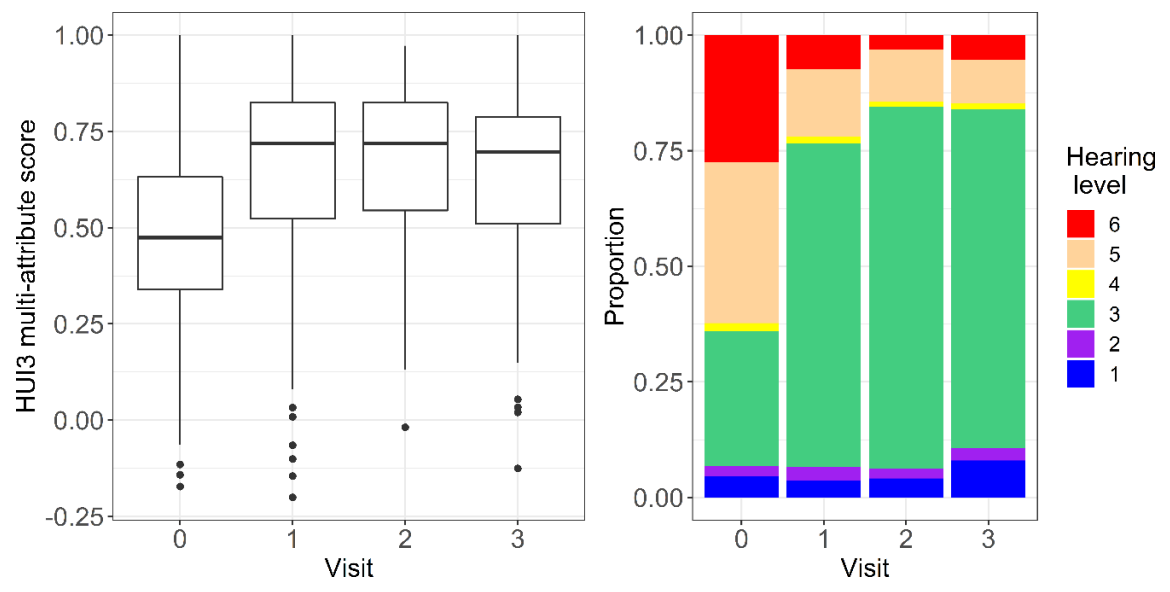